\title{
Characterizing the internal structure of laboratory ice samples with nuclear magnetic resonance
}

\author{
Timothy I. BROX, ${ }^{1 *}$ Mark L. SKIDMORE, ${ }^{2}$ Jennifer R. BROWN ${ }^{1}$ \\ ${ }^{1}$ Department of Chemical and Biological Engineering, Montana State University, Bozeman, MT, USA \\ ${ }^{2}$ Department of Earth Sciences, Montana State University, Bozeman, MT, USA \\ Correspondence: Jennifer R. Brown<jbrown@coe.montana.edu>
}

\begin{abstract}
Due to solute impurities and freezing-point depression in polycrystalline ice, a complicated and dynamic network of liquid water forms within the solid ice matrix at the boundaries between ice crystal grains. Impurity concentrations, temperature and pressure influence this network structure and impact physical, transport and rheological properties of ice. However, the nature of this internal network structure is not fully understood. Here we utilize nuclear magnetic resonance (NMR) measurements of diffusion and magnetic relaxation to study the geometry and interconnectivity of the liquid-filled network in laboratory ice, formed from a $7 \mathrm{~g} \mathrm{~L}^{-1} \mathrm{NaCl}$ solution, and its evolution due to recrystallization processes. Additionally, we apply these NMR measurements to observe the impact on ice microstructure of an ice-binding protein (IBP) excreted by the V3519-10 organism (Flavobacteriaceae family) isolated from the Vostok ice core in Antarctica. Recrystallization inhibition was observed as a function of IBP concentration. This work demonstrates the utility of advanced NMR techniques for applications to ice microstructure and has broader implications for understanding geophysical properties of cryospheric systems.
\end{abstract}

KEYWORDS: crystal growth, glaciological instruments and methods, ice biology, ice physics, recrystallization

\section{INTRODUCTION}

\subsection{Overview}

In the polycrystalline ice of glaciers, ice sheets and sea ice, the presence of impurities creates a freezing-point depression and liquid water gathers at junctions between solid ice crystal grains. At the grain boundaries of three ice crystals, there exists a network of liquid-filled veins, the cross sections of which are equilateral triangles with bowed-in faces (Nye and Frank, 1973; Nye, 1989; Mader, 1992). At two-grain boundaries, liquid is present in the planes between crystal faces (Cullen and Baker, 2001; Barnes and others, 2003; Barnes and Wolff, 2004; Mader and others, 2006; Grimm and others, 2008). Surface energy considerations have been used to describe the theoretical shape (Nye and Frank, 1973) and volume (Nye, 1989) of veins, and precise experimental work has been used to measure the geometry of enlarged veins (Mader, 1992). As ice ages, it undergoes recrystallization, a process in which larger ice crystals grow at the expense of smaller ones. This ripening process is driven by non-equilibrium thermodynamics to reduce the total interfacial energy of the system (Bray, 2002) and alters the dimensions of the liquid-filled junctions at ice grain boundaries.

Recently there has been an increased interest in the liquid-filled vein network within polycrystalline ice. Work has shown its importance in glacier rheology (Cuffey and others, 2000; West and others, 2007), contaminant transport (Jepsen and others, 2006) and as a potential microbial habitat (Price, 2000; Mader and others, 2006). Research has shown that microorganisms can remain viable (Priscu and

*Present address: MacDiarmid Institute for Advance Materials and Nanotechnology, School of Chemical and Physical Sciences, Victoria University of Wellington, Wellington, New Zealand. others, 1998, 1999; Skidmore and others, 2000; Christner and others, 2003) and even metabolically active (Christner, 2002; Bakermans and Skidmore, 2011a,b; Doyle and others, 2012) within solid ice at temperatures below $0^{\circ} \mathrm{C}$. One way in which freeze-tolerant microorganisms appear to have adapted to icy environments is via the production of icebinding proteins (IBPs) that bind to the surface of ice crystals and inhibit crystal growth. Proteins with ice-interacting properties have been studied extensively in organisms such as fish, cold hardy plants and insects, with functions ranging from ice nucleation to antifreeze and recrystallization inhibition (Ewart and others, 1999; Zachariassen and Kristiansen, 2000). Less is known about microbial IBPs, but they are produced by sea-ice diatoms, snow mold and certain sea-ice bacteria, with potential implications for geophysical properties of icy systems (Christner, 2010).

A protein produced by the bacterial strain V3519-10 (Flavobacteriaceae family), isolated from the Vostok ice core, was recently found to bind to prism faces of ice crystal grains and inhibit crystal growth along the a-axis, resulting in recrystallization inhibition activity (Raymond and others, 2008). The presence of IBPs increases bacterial viability during repeated freeze/thaw cycles (Achberger and others, 2011), suggesting that IBPs are an adaptive mechanism for microorganisms entrained in glacial ice to maintain the structure of the liquid-filled vein network, their proposed habitat.

Techniques that can non-destructively examine the threedimensional (3-D) network of liquid water collected at ice grain junctions are currently lacking. The liquid-filled veins at the intersection of three ice grain boundaries can be seen with conventional light microscopy or scanning electron microscopy; however, sample preparation procedures are destructive to the bulk sample and preclude the possibility of observing microstructural changes over time. Nuclear 
a

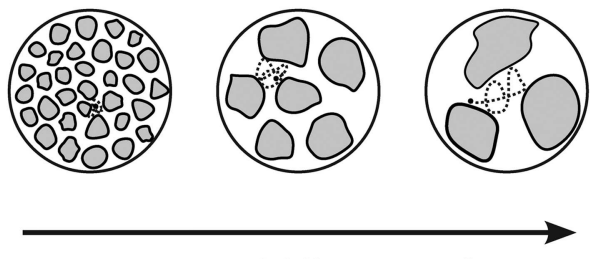

Increasing $T_{2}$ and diffusion coefficient b

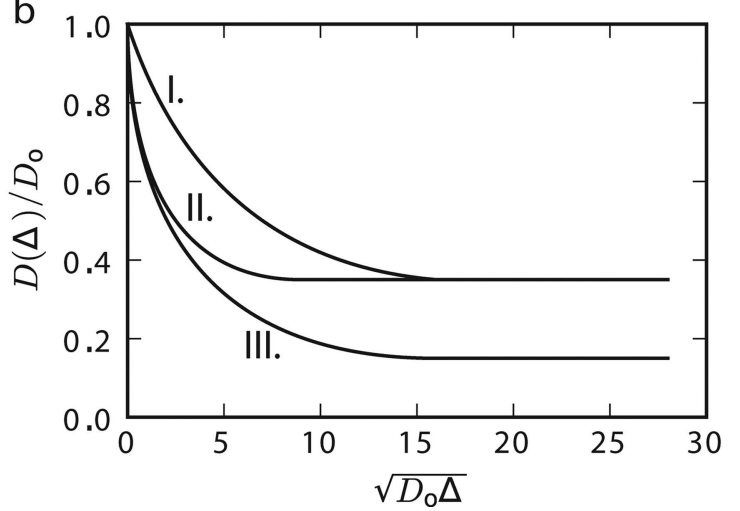

Fig. 1. (a) Schematic of restricted Brownian motion within a pore for a given observation time. (b) Schematic of time-dependent diffusion curves $D(\Delta)$ obtained from NMR PGSE measurements for idealized pore geometries, where $D_{\mathrm{o}}$ is the molecular self-diffusion coefficient of the unfrozen water and $\Delta$ is the observation time. Initial slopes give $S / V_{p}$ of the pore space, while the long time limit gives tortuosity, a measure of the network interconnectivity. I and II have the same tortuosity, but II has a larger $S / V_{\mathrm{p}}$. II has a low tortuosity, while III is more restricted. Reproduced from Sen (2004).

magnetic resonance (NMR) is a non-invasive and nondestructive technique that can provide a wide range of microstructural information about the network of veins and planar junctions, including water content, visualization of vein network structure, quantitative surface-to-volume ratios, and interconnectivity of the network.

Previous research has used NMR techniques to measure liquid water content in ice (Richardson and Keller, 1966; Richardson, 1976; Grimm and others, 2008; Mel'nichenko and Slobodyuk, 2013) and ice/silicate mixtures (Stillman and others, 2010) as well as to image distribution of liquid water (Edelstein and Schulson, 1991; Menzel and others, 2000) in sea ice. However, NMR measurements of relaxation rates and time-dependent molecular diffusion, used extensively to probe pore structure and transport dynamics in porous media systems (Hurlimann and others, 1994; Song and others, 2008), is a novel approach for characterizing the internal structure of ice (Brown and others, 2012, 2014). In this work, we use these NMR techniques to investigate the impact of microbial V3519-10 IBP on the liquid vein network structure and recrystallization processes in laboratory-made ice.

\subsection{Nuclear magnetic resonance}

The liquid state ${ }^{1} \mathrm{H}$ NMR signal is a direct measure of the hydrogen nuclei spin density and therefore the quantity of water present in an ice sample (Richardson and Keller, 1966; Richardson, 1976). The signal can be used to calculate the volume of unfrozen water in the ice sample once referenced to the signal from a known water volume for a given receiver gain, and adjusting for the Boltzmann distribution signal dependence on temperature.

Magnetic resonance imaging $(\mathrm{MRI})$ is a common NMR technique in medical applications and can be applied to any system with NMR-active nuclei, such as the ${ }^{1} \mathrm{H}$ nuclei associated with water. Therefore, standard imaging techniques can be used to visualize the liquid vein network in ice (Edelstein and Schulson, 1991; Menzel and others, 2000). However, depending upon impurity concentration and temperature, vein dimensions can be smaller than the practically achievable spatial resolution due to a low NMR signal-to-noise ratio and the inherent water diffusion limit (Callaghan, 1991). In these cases, one can utilize NMR techniques developed for probing pore structure and transport dynamics in porous media via measurement of relaxation rates and molecular diffusion (Callaghan and others, 1991; Mitra and others, 1992; Song and others, 2008). NMR magnetic relaxation time and molecular diffusion measurements are sensitive to the dimensions of liquid-filled junctions between three and two ice grains (Brown and others, 2012), giving quantitative information about the network structure and providing a novel approach for monitoring ice microstructure and its evolution with time.

Spin-spin $\left(T_{2}\right)$ magnetic relaxation time measurements have been used extensively to probe pore structure in porous media (Hurlimann and others, 1994; Mair and others, 1999; Song and others, 2008), where the geometry of a liquid-filled pore restricts translational or rotational mobility of nuclear spins. The amplitude of $T_{2}$ relaxation times is proportional to the surface-area-to-volume ratio within the confining space, scaling as (Brownstein and Tarr, 1979)

$$
1 / T_{2} \sim \rho S / V_{\mathrm{p}}
$$

where $S$ is pore surface area, $V_{p}$ is pore volume, and the constant of proportionality $\rho$ is the surface relaxivity. In the case of ice, the 'pores' are the liquid-filled veins located at three-grain junctions and the two-grain planar junctions.

The proportionality in Eqn (1) holds in the regime where diffusional mixing of the surface and bulk fractions is faster than the difference in intrinsic relaxation rates, as is valid for the ice studied here. Thus comparatively shorter $T_{2}$ measurements indicate a larger $S / V_{\mathrm{p}}$, i.e. a more restricted environment and therefore smaller vein dimensions (Fig. 1a). $T_{2}$ times are measured from the signal arising from the entire volume of the ice sample and therefore represent an average over the 3 -D pore space. These measurements have the advantage of rapid acquisition, on the order of seconds to minutes, as opposed to hours for imaging.

With pulsed-gradient spin-echo (PGSE) NMR, the mean squared displacement of an ensemble of spins may be measured directly (Caprihan and Fukushima, 1990; Callaghan, 1991). This mean squared displacement is related to the diffusion coefficient of the confined liquid water, the magnitude of which depends upon the geometry and size of the confining pore. For unrestricted Brownian motion in 3-D, the mean squared displacement of a molecule grows linearly with time in a relation first introduced by Einstein,

$$
\left\langle\left[r^{\prime}(\Delta)-r(0)\right]^{2}\right\rangle=6 D_{o} \Delta
$$


Here $r$ and $r^{\prime}$ are position vectors at times zero and $\Delta$, respectively, and $D_{o}$ is the constant molecular self-diffusion coefficient. In restricted diffusion, the effective diffusion coefficient decreases with increasing observation time in a manner depending upon the restricting geometry (Mitra and others, 1993).

Based on the model of diffusion as random walkers, the long and short time limits of the time-dependent diffusion coefficient in relation to the pore space in a well-connected porous medium can be calculated (Mitra and others, 1993; Sen, 2004). In the short time limit, a fraction of the liquid molecules in the pore space interact with the boundaries of the solid matrix, giving rise to a time-dependent diffusion coefficient proportional to $S / V_{p}$

$$
\frac{D(\Delta \rightarrow 0)}{D_{o}}=1-\frac{4}{9 \sqrt{\pi}} \frac{S}{V_{\mathrm{p}}} \sqrt{D_{o} \Delta}
$$

In the long time limit, diffusion in a well-connected medium approaches a non-zero value,

$$
\frac{D(\Delta \rightarrow \infty)}{D_{o}}=\frac{1}{\alpha}
$$

The normalized diffusion coefficient is reduced from free diffusion by a geometric factor termed tortuosity, $\alpha$. Tortuosity is the ratio of the path length a molecule travels in a porous medium to the geometric length traversed, $\alpha=I_{\text {path }} / I_{\text {geom, }}$ and is a measure of interconnectivity of the pore space (Sen, 2004). The distances a molecule needs to diffuse to reach this asymptotically long time limit are typically longer than accessible with NMR due to spin-lattice $\left(T_{1}\right)$ relaxation decreasing the NMR signal. In this case, a Padé approximation can be used to interpolate between the short and long time regimes (Latour and others, 1993):

$$
\frac{D(\Delta)}{D_{o}}=1-\left(1-\frac{1}{\alpha}\right)\left(\frac{\frac{4}{9 \sqrt{\pi}} \frac{S}{V_{\mathrm{p}}} \sqrt{D_{o} \Delta}+\left(1-\frac{1}{\alpha}\right) \frac{D_{o} \Delta}{D_{o} \theta}}{\left(1-\frac{1}{\alpha}\right)+\frac{4}{9 \sqrt{\pi}} \frac{S}{V_{\mathrm{p}}} \sqrt{D_{o} \Delta}+\left(1-\frac{1}{\alpha}\right) \frac{D_{o} \Delta}{D_{o} \theta}}\right)
$$

This form includes a fitting parameter $\theta$ that represents the time for a particle to diffuse the distance needed to reach the tortuosity limit. By fitting experimental data to this model, it is possible to extract approximate values for tortuosity, despite the lack of coherent NMR signal at long observation times. This approximation has been tested on various model bead packs and found to provide good estimations of $S / V_{p}$ and tortuosity (Mair and others, 1999, 2001). Figure $1 \mathrm{~b}$ shows representative examples of the time-dependent diffusion curves, demonstrating how differences in $S / V_{p}$ and tortuosity would manifest.

\section{METHODS}

\subsection{Sample preparation}

Five ice samples were prepared. All samples contained a $7 \mathrm{~g} \mathrm{~L}^{-1} \mathrm{NaCl}$ solution, which is a solute concentration comparable with measurements in Antarctic basal ice (Montross and others, 2014). Three concentrations of IBP were studied: $10 \mu \mathrm{g} \mathrm{mL}^{-1}$ of V3519-10 naturally secreted extracellular proteins (ECPs; $>30 \mathrm{kDa}$ with an unknown IBP fraction); and purified recombinant IBP (rIBP) at 2 and $4 \mu \mathrm{g} \mathrm{mL}^{-1}$ concentrations. Two control samples were prepared: a control consisting of $7 \mathrm{~g} \mathrm{~L}^{-1} \mathrm{NaCl}$ solution without protein and a sample of $7 \mathrm{~g} \mathrm{~L}^{-1} \mathrm{NaCl}$ solution containing the protein bovine serum albumin (BSA) at $10 \mu \mathrm{g} \mathrm{mL}^{-1}$ concentration.
The ice with BSA was used to examine any impact on ice microstructure from colligative effects due to the presence of a non-ice-binding macromolecule. BSA was chosen as it is a similar size $(\sim 64 \mathrm{kDa})$ to the V3519-10 IBP $(\sim 52 \mathrm{kDa})$, but does not exhibit ice-binding activity. BSA standards were purchased (Thermo Scientific No. 23209) and used without further purification. To obtain V3519-10 ECPs, the V3519-10 bacteria were grown in R2 broth (Atlas, 1995) at $4{ }^{\circ} \mathrm{C}$ until the culture reached an optical density $\mathrm{OD}_{595}$ of 0.22 , at which time it was centrifuged at $5000 \mathrm{~g}$ for $30 \mathrm{~min}$ at $4{ }^{\circ} \mathrm{C}$ to pellet the cells and recover the supernatant. The supernatant containing the IBP was filtered using Amicon Ultra-15 centrifugal filters with a nominal threshold of $30 \mathrm{kDa}$ to obtain a crude extract of V3519-10 ECP. Recombinant IBP was provided by colleagues at Louisiana State University, Baton Rouge, LA, USA. The protein concentration of samples frozen in the presence of BSA, V3519-10 ECP and recombinant V3519-10 IBP was determined by a Bradford assay (Thermo Scientific No. 23236) prior to freezing.

All samples were prepared by filling $13 \mathrm{~mm}$ outer diameter (11.7 mm internal diameter) standard NMR tubes with $8 \mathrm{~mL}$ of solution, placing them in a polystyrene sample holder, insulated on the sides and bottom, and freezing them in a Revco ULT-750 chest freezer at $-13.5^{\circ} \mathrm{C}$. As the tube walls were rigid, the bulk ice sample elongated due to expansion of the ice. This was believed to cause stress within the ice at the bottom of that sample. To prevent these artifacts from affecting the measured structure, the samples were oriented within the NMR system so that the only signal detected came from a $\sim 20 \mathrm{~mm}$ tall slice in the center of the ice column. Multiple samples of each concentration were frozen and, as determined by MR imaging and by eye, ice samples that were cloudy or contained air bubbles were discarded as supercooling and subsequent rapid freezing may have occurred. Samples were transferred from the chest freezer in a cooler filled with gel freezer packs stored in the same freezer. Transfer time of the ice from the cooler to the NMR spectrometer was minimized to $\sim 3 \mathrm{~min}$. The NMR magnet electronics were always pre-cooled at the set temperature before sample insertion and the set temperature equilibrated within $\sim 1 \mathrm{~min}$ after loading a sample. The samples were allowed to equilibrate at the set temperature for $45 \mathrm{~min}$ before measurements were performed. Samples were analyzed via NMR at multiple time points over 1800 hours $\left(\sim 11\right.$ weeks), and stored in the freezer at $-13.5^{\circ} \mathrm{C}$ between NMR measurements.

\subsection{NMR methods}

NMR measurements were performed on a Bruker DRX250 spectrometer with a $5.8 \mathrm{~T}$ superconducting vertical widebore magnet and Micro2.5 gradient imaging probe. The samples were centered in a $20 \mathrm{~mm}$ diameter radio-frequency (RF) coil using a PEEK spacer to allow for air flow around the sample. Sample temperature was controlled by the flow of cooled nitrogen gas, the temperature of which was regulated by a thermocouple at the base of the sample. The set point temperature for the base of the sample was $-16^{\circ} \mathrm{C}$. The temperature of airflow at the top of the sample was measured during experiments and was typically $-13^{\circ} \mathrm{C}$ for a temperature increase of $3^{\circ} \mathrm{C}$ across the $70 \mathrm{~mm}$ long RF coil.

Unfrozen water content was calculated from the NMR signal magnitude after calibration with a known volume of water measured as a function of temperature for a fixed receiver gain. Any signal from the solid ice crystals was not 


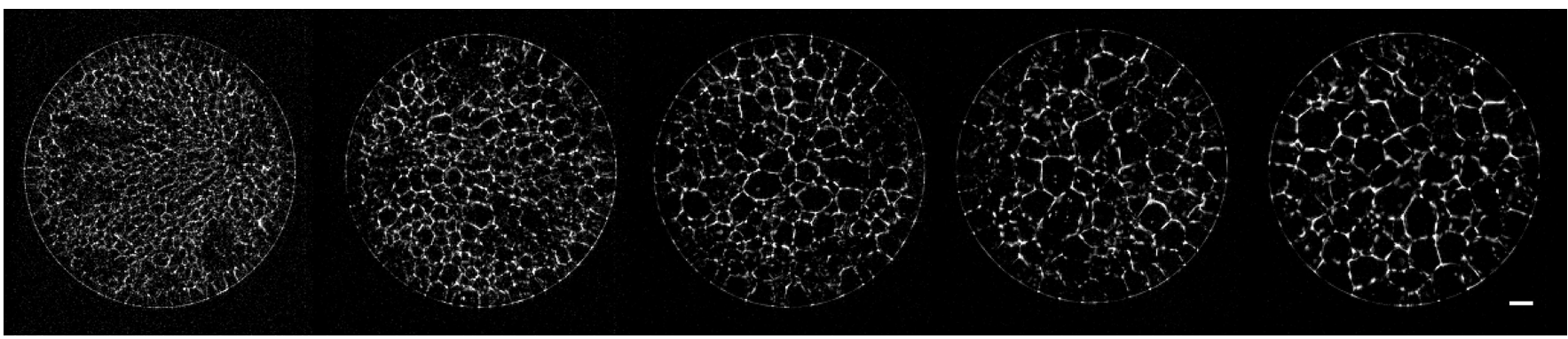

Fig. 2. Cross-sectional images of ice frozen in the presence of BSA. Age of ice (left to right) 39, 259, 578, 938 and 1705 hours. Spin-echo images with $55 \mu \mathrm{m} \times 55 \mu \mathrm{m}$ spatial resolution in the $x-y$ plane; 172 averages; FOV $14 \mathrm{~mm} \times 14 \mathrm{~mm}$; matrix $256 \times 256$; slice thickness $0.5 \mathrm{~mm}$. Scale bar is $1 \mathrm{~mm}$.

detectable. The NMR signal was from liquid water only, i.e. no solid-state Gaussian signal from the ice phase was detected due to the RF excitation and signal acquisition digitization timescales. Cross relaxation between the solid ice crystal phase and liquid water in veins can be neglected based on this and the large difference between the water diffusivity $\left(\sim 10^{-10} \mathrm{~m}^{2} \mathrm{~s}^{-1}\right)$ and the spin diffusion $\left(\sim 10^{-15} \mathrm{~m}^{2} \mathrm{~s}^{-1}\right)$ (Valiullin and Furó, 2002). Measurements of NMR signal magnitude were collected frequently during an ice sample's time in the magnet, serving as a check for melting within the sample. $T_{2}$ relaxation time distributions were obtained using a standard Carr-Purcell-Meiboom-Gill (CPMG) echo train, with echo spacing $t_{\mathrm{E}}=403 \mu \mathrm{s}$. A standard pulsed-gradient stimulated-echo (PGSTE) sequence was used to measure diffusion for displacement observation times $\Delta$ ranging from 10 to $1000 \mathrm{~ms}$ at a constant echo time $t_{\mathrm{E}}$ of $8 \mathrm{~ms}$ and gradient duration $\delta=2 \mathrm{~ms}$. Diffusion coefficients were calculated from a standard StejskalTanner plot and the fit was linear, with no indication of multiexponential decay. The mono-exponential decay was also confirmed by performing an inverse Laplace transform, which resulted in a single diffusion coefficient. Images were obtained with a standard two-dimensional (2-D) multi-slice spin-echo sequence and had a spatial resolution of $55 \mu \mathrm{m}$ $\times 55 \mu \mathrm{m}(256 \times 256$ matrix size and $14 \mathrm{~mm} \times 14 \mathrm{~mm}$ field of view (FOV)) over a $0.5 \mathrm{~mm}$ thick slice centered in the middle of the RF coil.

\subsection{Curve fitting}

A Padé approximation fit (Eqn (5)) was performed to extract ice microstructural parameters, including $S / V_{\mathrm{p}}$ and $\alpha$, from the time-dependent diffusion data. Each series of diffusion coefficient data as a function of observation time $D(\Delta)$ was fit using a Python script utilizing the Imfit package (version 0.5 ) for least-squares minimization with constraints. The optimization method used by the Imfit package was the Levenberg-Marquart algorithm.

The free diffusion coefficient $D_{0}$ was fixed at $5.6 \times$ $10^{-10} \mathrm{~m}^{2} \mathrm{~s}^{-1}$ for each dataset. This value was estimated by extrapolating the time-dependent diffusion data at short observation times (up to $\Delta=50 \mathrm{~ms}$ ) back to zero. The resulting $D_{O}$ is consistent with the diffusion coefficient expected when taking into account the effect of salt concentration (Kim and Yethiraj, 2008) and temperature (Price and others, 1999). For $D_{o}=5.6 \times 10^{-10} \mathrm{~m}^{2} \mathrm{~s}^{-1}$, a known temperature of $-13.5^{\circ} \mathrm{C}$ and measured water content of $2.4 \%$, the corresponding salt concentration in the unfrozen brine is expected to be $4 \mathrm{M} \mathrm{NaCl}$ (Price and others, 1999; Kim and Yethiraj, 2008). If it is assumed that all salt is partitioned into the liquid water upon freezing, the expected salt concentration is $5 \mathrm{M}$. This indicates that some salt is likely being partitioned into the solid ice matrix, as has been reported for ice within the Dome Fuji (Antarctica) ice core (Ohno and others, 2005). The surface-to-volume ratio $S / V_{\mathrm{p}}$ parameter was determined by linearly fitting diffusion data in the short time regime (Eqn (3)). The points used in this fit included diffusion measurements from the two shortest observation times ( $\Delta=15$ and $20 \mathrm{~ms}$ ) in addition to a point at $t=0$ where the diffusion coefficient would be equal to the free unrestricted diffusion coefficient (Eqn (3)).

With the free diffusion coefficient $D_{o}$ fixed and the surface-to-volume ratio determined from the short time behavior, two fitting parameters, $\alpha$ and $\theta$, remained for the Padé approximation (Eqn (5)). The same initial values for the two fitting parameters were used for all datasets $\left(\alpha_{\text {initial }}=5 \mathrm{~s}\right.$; $\theta_{\text {initial }}=0.1 \mathrm{~s}$ ). Owing to NMR signal loss from $T_{1}$ relaxation, observation times in the asymptotically long time limit are not accessible with ${ }^{1} \mathrm{H}$ NMR for the ice samples and therefore the fitting results in an effective $\alpha$. The Padé fit parameters are highly sensitive to the chosen value of $D_{o}$.

\section{RESULTS}

Magnetic resonance images were collected on ice samples as they aged. Cross-sectional images acquired for the ice frozen in the presence of BSA at various time points after freezing are shown in Figure 2. The resolution of the images is $55 \mu \mathrm{m} \times 55 \mu \mathrm{m}$ in the $x-y$ plane and the images are averaged over a $0.5 \mathrm{~mm}$ thick slice in the $z$ dimension. Multiple $0.5 \mathrm{~mm}$ thick cross-sectional slices were acquired along the height of each sample over the $\sim 20 \mathrm{~mm}$ NMR active region in the RF coil. All the images presented here are from the slice located approximately at the center of the active region. Light regions of the images correspond to liquid water in the veins, while dark regions indicate a lack of signal and therefore solid ice crystals. The veins or threegrain junctions are clearly visible; however, the thin plane features located between two crystal faces are not distinguishable as their dimensions are below the $55 \mu \mathrm{m}$ spatial resolution. The crystals are small $(\sim 200 \mu \mathrm{m})$ soon after initial freezing ( $t \sim 100$ hours). As the ice ages over 1600 hours, the images indicate definitive crystal growth, or coarsening, with crystal diameters growing to $\sim 1 \mathrm{~mm}$. The sample frozen without added proteins had a similar structure and the crystal fabric also coarsens (Fig. 2). With the growth of ice crystals there is a corresponding growth in vein dimensions.

This is consistent with a quantitative model in which veins are treated as ideal cylinders and ice crystal grains are 


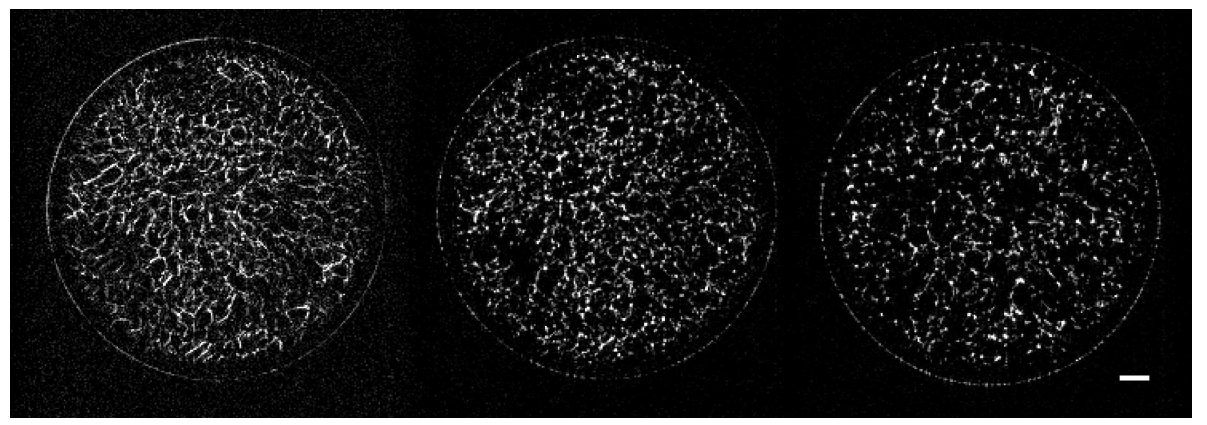

Fig. 3. Cross-sectional images of ice containing $10 \mu \mathrm{g} \mathrm{mL}^{-1} \mathrm{~V} 3519-10$ ECP. Age of ice (left to right) 102, 651 and 1730 hours. Spin-echo images with $55 \mu \mathrm{m} \times 55 \mu \mathrm{m}$ spatial resolution in the $x-y$ plane; 172 averages; FOV $14 \mathrm{~mm} \times 14 \mathrm{~mm}$; matrix $256 \times 256$; slice thickness $0.5 \mathrm{~mm}$. Scale bar is $1 \mathrm{~mm}$.

semiregular truncated octahedrals (Price, 2000). In this model, the vein diameter found along the line where three ice crystals meet, $d_{\text {vein, }}$ can be estimated as (Grimm and others, 2008)

$$
d_{\text {vein }}=0.3874 D \sqrt{\phi_{\mathrm{w}}}
$$

where $D$ is the crystal diameter and $\phi_{\mathrm{w}}$ is the volume fraction of unfrozen water. The planar two-grain junctions have a diameter of

$$
d_{\text {plane }}=0.2987 D \sqrt{\phi_{\mathrm{w}}}
$$

The geometric mean of these two models is

$$
d_{\text {mean }}=0.34 D \phi_{\mathrm{w}}^{3 / 4}
$$

and can be taken as representative of an average liquidfilled channel dimension in the ice. With the volume fraction of unfrozen water fixed, as ice crystals grow so must the cross section of the liquid-filled veins and planar junctions.

In contrast, ice with ECP (Fig. 3) exhibited static crystal structure, with a smaller and more randomized vein network structure that persisted with ice aging, ostensibly due to IBP binding to the ice crystal surface inhibiting crystal growth (Raymond and others, 2008). While the images clearly indicate differences in the two samples, limited spatial resolution precludes quantitatively calculating the vein size distribution or visualizing the two-grain planar junctions. In addition, the vein dimensions of the ice samples frozen in the presence of recombinant IBP were smaller than the MRI spatial resolution and so could not be discerned in images. Therefore, other NMR methods for additional characterization of the vein network structure were needed. NMR relaxation and time-dependent diffusion data provide a host of quantitative information on the liquid-filled network structure in addition to imaging and provide a valuable alternative when imaging is not possible or as a complement to visualization through imaging.

The water content in ice is a quantity of significant interest in geophysical applications, but without magnetic resonance techniques is often difficult to measure. Figure 4 shows volume fraction of unfrozen water as measured from the NMR signal intensity. All samples had similar water content $(\sim 2.4 \%)$ and this value did not change significantly as the samples aged. The measured liquid water fraction from NMR is slightly lower than the $\sim 2.9 \%$ predicted by the equilibrium thermodynamic model FREZCHEM, shown as the dashed line in Figure 4 (Marion and others, 2010). This model uses the Pitzer approach for writing activity coefficients with a temperature dependence and then minimizes the Gibbs free energy of the entire system (Marion and others, 2010).

NMR PGSE provides microstructural information on the network of liquid-filled junctions between ice crystal grains (i.e. $S / V_{\mathrm{p}}$ and $\alpha$ ) through measurement of an observationtime-dependent effective diffusion coefficient $D(\Delta)$. When water is confined within the grain junctions and is interacting with the solid ice crystals, diffusion becomes restricted and depends upon the geometry and size of the confining space (Mitra and others, 1993). For a given ice sample age, the effective diffusion coefficient is measured multiple times for increasing displacement observation time $\Delta$, and changes in the amplitude of $D(\Delta)$ reveal pore space structural characteristics (Mitra and others, 1993; Sen, 2004).

In the short displacement time, the time-dependent diffusion coefficient normalized by molecular diffusion $D(\Delta) / D_{o}$ is proportional to $S / V_{\mathrm{p}}$ (Eqn (3)) due to interaction of liquid molecules in the pore space with boundaries of the solid matrix (Sen, 2004). In the long displacement time, $D(\Delta) / D_{o}$ asymptotes to $1 / \alpha$ (Eqn (4)), where $\alpha$ is geometric tortuosity, a measure of interconnectivity of the pore space (Sen, 2004). Here we are limited to observing the approach to asymptotic diffusion (out to $\Delta \sim 1000 \mathrm{~ms}$ ) due to NMR

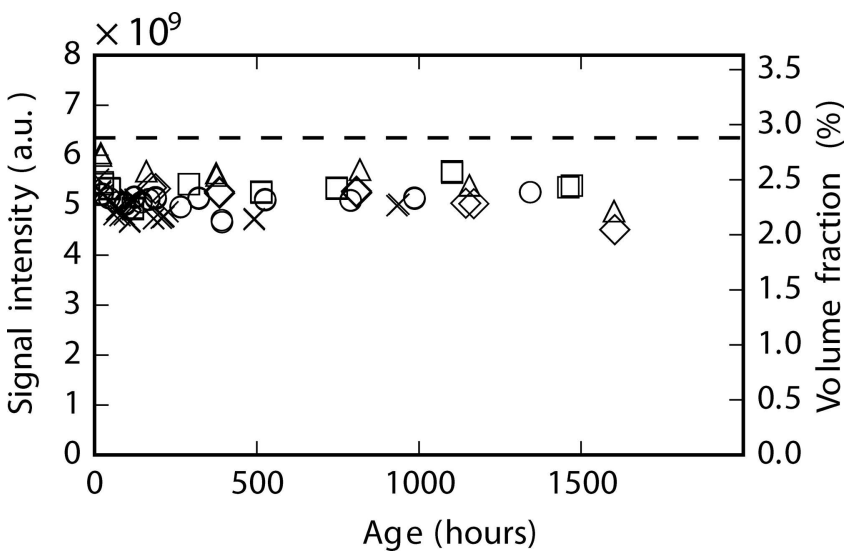

Fig. 4. NMR signal intensity and corresponding water volume fraction for all ice samples as a function of age. Ice control with $7 \mathrm{~g} \mathrm{~L}^{-1} \mathrm{NaCl}$ (circles), ice with $10 \mu \mathrm{g} \mathrm{mL}^{-1} \mathrm{BSA}$ (squares), ice with $10 \mu \mathrm{g} \mathrm{m}^{-1}$ V3519-10 ECP (crosses), ice with $2 \mu \mathrm{g} \mathrm{mL}^{-1} \mathrm{rlBP}$ (diamonds) and ice with $4 \mu \mathrm{g} \mathrm{mL}^{-1} \mathrm{rIBP}$ (triangles). The dashed line shows the volume fraction of unfrozen water predicted by the FREZCHEM geochemical model. 

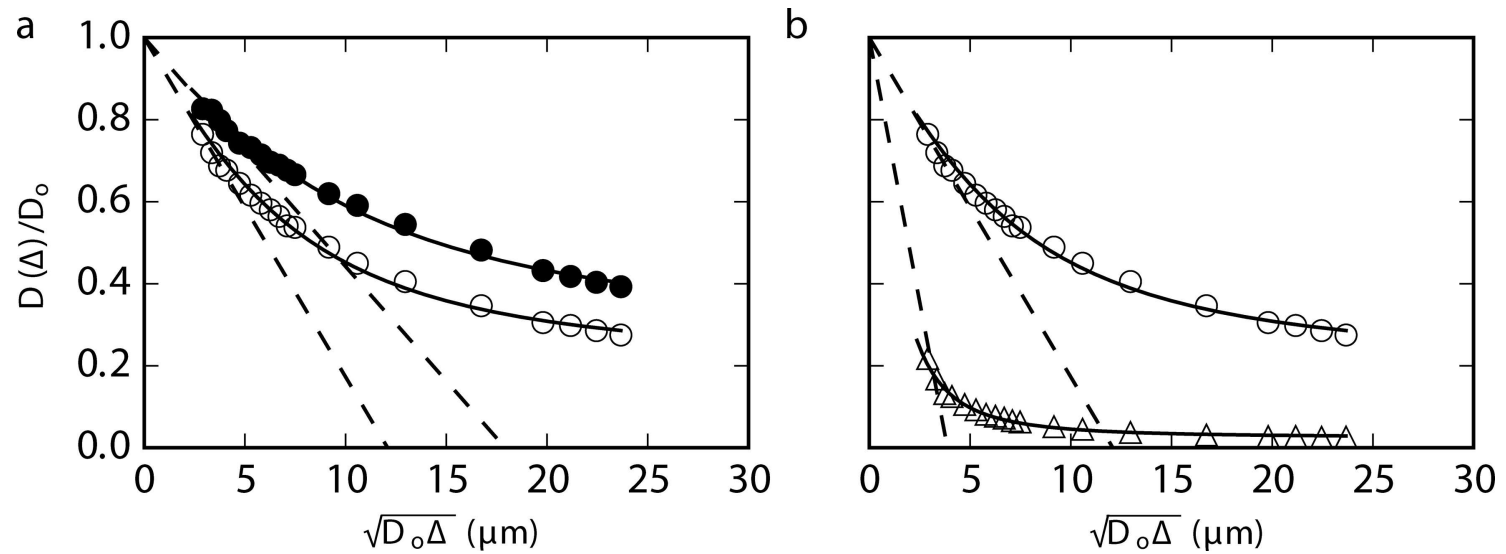

Fig. 5. Representative example of PGSTE diffusion measurements as a function of observation time. Padé fit is drawn with a solid line. Dashed lines are the linear short time regime, where the slope represents the surface-to-volume ratio. The free diffusion coefficient was fixed and estimated at $5.6 \times 10^{-10} \mathrm{~m}^{2} \mathrm{~s}^{-1}$ based on extrapolation of the short observation time data to zero, and is consistent with the value expected considering salt concentration (Kim and Yethiraj, 2008) and temperature (Price and others, 1999). (a) Evolution of the control sample as a function of ice aging. Open circles are the control sample at 46 hours and closed circles are the control sample at 987 hours. (b) Comparison between the control sample at 46 hours (open circles) and ice with $4 \mu \mathrm{g} \mathrm{mL}^{-1} \mathrm{rlBP}$ at 20 hours (open triangles) demonstrating the impact of ice-binding activity on the time-dependent diffusion curves.

signal loss via $T_{1}$ magnetic relaxation and therefore measure an effective $\alpha$.

Figure 5 displays representative examples of the timedependent diffusion curves for the ice control and $4 \mu \mathrm{g} \mathrm{mL}^{-1}$ rIBP samples. Effective diffusion coefficient measurements were made at 20 values of $\Delta$ between 10 and 1000 ms. Each set of 20 diffusion measurements was fit to a Padé curve (as described in Section 2.3). Figure 5a illustrates how the diffusion curve of the ice control evolves as ice crystals grow with aging, while Figure $5 \mathrm{~b}$ shows the difference in the curves between the ice control (larger crystals and veins) and rIBP (smaller crystals and veins) at a similar age. Symbols are experimental data, solid curves are the Padé fit and dashed curves are the fit to the short time decay in the diffusion coefficient, resulting in the $S / V_{\mathrm{p}}$. The $S / V_{\mathrm{p}}$ from the short time fit, and the fitting parameters $\theta$ and tortuosity $\alpha$ from the Padé fit, are plotted for all samples as a function of age in Figure 6a-c, respectively.

As can be seen in Figure 6a, there is an evolution of $S / V_{p}$ with age in the ice control and ice with BSA samples, i.e. $S / V_{p}$ decreased with time. Since the total volume of liquid water did not change with age (Fig. 4), the vein volume $V_{p}$ was fixed and therefore the surface area of the ice crystals in contact with the liquid decreased. Modeling the veins at three-grain junctions as a cylinder (Price, 2000), the $S / V_{\mathrm{p}}$ can be calculated as $4 / d_{\text {vein. }}$. The two-grain planar junctions have an $S / V_{p}$ of $1 / d_{\text {plane. }}$ The measured $S / V_{p}$ represents an average of water confined within the three- and two-grain junctions, which we will define here as $d_{\text {ave }}=V_{\mathrm{p}} / S$. For the ice with BSA, $S / V_{p}$ decreases from $0.36 \mu \mathrm{m}^{-1}$ at $t=27$ hours to $0.14 \mu \mathrm{m}^{-1}$ at $t=1104$ hours. This results in a change in $d_{\text {ave }}$ from $2.8 \mu \mathrm{m}$ at short aging times to $7.1 \mu \mathrm{m}$ at long aging times. Using the image in Figure 2 at $t=938$ hours to obtain a rough estimate of crystal size $(D=750 \mu \mathrm{m}), d_{\text {mean }}$ is calculated to be $\sim 16 \mu \mathrm{m}$ (Eqn (8)), $d_{\text {vein }} \sim 46 \mu \mathrm{m}$ (Eqn (6)) and $d_{\text {plane }} \sim 5 \mu \mathrm{m}$ (Eqn (7)). We can see that our measured $S / V_{\mathrm{p}}$ returns a dimension $\left(d_{\mathrm{ave}}=V_{\mathrm{p}} / S=7.1 \mu \mathrm{m}\right)$ that is on the same order of magnitude as expected based upon the crystal sizes seen in the images $\left(d_{\text {mean }}=16 \mu \mathrm{m}\right)$, with a possible weighting towards the smaller two-grain planar junctions $\left(d_{\text {plane }}=5 \mu \mathrm{m}\right)$. However, it should be noted that the in-plane image resolution $(55 \mu \mathrm{m} \times 55 \mu \mathrm{m})$ and the averaging over the slice thickness in the $z$-dimension $(0.5 \mathrm{~mm})$, as well as the visible size distribution of ice crystals, precludes obtaining reliably quantitative crystal sizes. Therefore, the $S / V_{\mathrm{p}}$ measurement is a better approach for obtaining quantitative results.

The ice control showed very similar behavior in $S / V_{\mathrm{p}}$ demonstrating that proteins without ice-binding activity have no impact on the ice structure and thus the network of liquid-filled grain junctions. While time-dependent diffusion data only exist at a few time points for the ice containing $E C P$, it can be seen in Figure 6a that $S / V_{\mathrm{p}}$ decreases with aging (from $0.45 \mu \mathrm{m}^{-1}$ at $t=92$ hours to $0.34 \mu \mathrm{m}^{-1}$ at $t=933$ hours), but to a lesser degree than is observed in the ice control. These $S / V_{\mathrm{p}}$ values correspond to an average crystal junction size $d_{\text {ave }}$ of 2.2 and $2.9 \mu \mathrm{m}$. In the sample frozen in the presence of $2 \mu \mathrm{g} \mathrm{mL}^{-1} \mathrm{rlBP}, S / V_{\mathrm{p}}$ changes only slightly from 0.72 to $0.68 \mu \mathrm{m}^{-1}$ for $t$ from 189 to 810 hours, resulting in a negligible change in $d_{\text {ave }}$. For the $4 \mu \mathrm{gL}^{-1} \mathrm{rIBP}$ sample, $S / V_{\mathrm{p}}$ is $1.0 \mu \mathrm{m}^{-1} \quad\left(d_{\mathrm{ave}}=1 \mu \mathrm{m}\right)$ and does not change with aging, indicating a static crystal structure and liquid-filled grain junction size by the time the first diffusion curve was collected ( $t=20$ hours). Potentially, recrystallization could occur at times $<20$ hours after freezing. Most likely, each sample begins with the same structure, but is driven to recrystallize and minimize the surface energy interactions of the ice-ice and ice-vein interfaces. In the samples with the rIBP, the action of these proteins binding to an ice crystal face inhibits crystal growth, preventing the system from seeking a lower energy state. The $S / V_{\mathrm{p}}$ is clearly dependent upon IBP concentration, with increasing protein resulting in smaller and more persistent crystals.

Although imaging was not possible for ice frozen with rIBP, these data suggest that the rIBP was able to prevent the liquid network from changing with age due to recrystallization inhibition. Previous work has already demonstrated that natural V3519-10 ECPs inhibit crystal growth (Raymond and others, 2008) and it has been suggested that these IBP are a mechanism to control the vein network as a microbial habitat. However, to our knowledge, there is currently no research demonstrating that these proteins maintain the vein 
network structure for extended time periods and thus act as a potential survival mechanism. Here we see persistent small crystal structure up to 1600 hours.

The ice control and ice with BSA both clearly exhibit $S / V_{\mathrm{p}}$ that change with time. Recrystallization is a thermodynamically driven coarsening, often considered a form of Ostwald ripening where the solid phase, modeled as spherical particles, maintains a constant volume fraction. According to the theory of Lifshitz, Slyozov and Wagner (LSW theory) for Ostwald ripening (Lifshitz and Slyozov, 1961; Wagner, 1961), the crystal size increases with a dependence on time of $t^{1 / 3}$ in 3-D (Kahlweit, 1975). However, fitting a power-law function to $V_{p} / S$ (the inverse of $S / V_{p}$ and a length scale proportional to vein size and therefore crystal size) reveals a time dependence of $\sim t^{1 / 7}$. LSW theory was developed for low volume fractions of the dispersed phase, in this case the solid ice crystals. The solid phase here however is at high volume fractions, $\sim 0.98$.

Modifications to the LSW theory (Brailsford and Wynblatt, 1979; Voorhees and Glicksman, 1984; Stevens and Davies, 2002) have addressed ripening at finite volume fractions, but predict an increase of the rate kinetics, as opposed to altered time dependence. However, another mechanism of crystal growth that becomes important at high volume fractions, where crystals are in close proximity in space, is accretion. In accretion, two crystals fuse into a single larger aggregate crystal. This could be the dominant mechanism of recrystallization in these low-porosity samples, causing a smaller dependence on time, especially at shorter freezing times.

Figure $6 \mathrm{~b}$ and $\mathrm{c}$ shows the Padé fitting parameter $\theta$ and effective tortuosity, a measure of interconnectivity of the liquid-filled grain junctions, respectively. For all samples, the effective tortuosity stays relatively constant; however, the fitting parameter $\theta$ trends in a similar manner to $S / V_{p}$. Since $\theta$ represents the time for a particle to diffuse the distance needed to reach the tortuosity limit, it can be expected to be very sensitive to crystal size. This is indeed observed because with larger crystals or as the crystals grow, $\theta$ increases in the same manner as the $S / V_{\mathrm{p}}$ ratio decreases. The average effective tortuosity was lowest for the ice frozen with BSA $(\sim 3.6)$ and ice frozen without added proteins $(\sim 4.3)$. For the ice with $\mathrm{ECP}$, average effective tortuosity is only slightly larger than for the ice control $(\sim 6.7)$, but in the rIBP samples average $\alpha$ increases significantly over the ice control. Average $\alpha$ is $\sim 12.6$ for the ice containing $2 \mu \mathrm{g} \mathrm{mL}^{-1}$ rIBP, while for ice with $4 \mu \mathrm{g} \mathrm{mL}^{-1}$ rIBP it is ten times that of the ice control, at 49. These data indicate that with increasing IBP concentration, crystals remain small and the path a molecule must follow to diffuse through the liquid-filled network becomes more 'tortuous', hence larger values of effective $\alpha$.

Spin-spin relaxation times $\left(T_{2}\right)$ were measured for all samples as a function of ice aging (Fig. 7). Figure 7a shows an increase in $T_{2}$ values for the ice with BSA and ice frozen without added proteins, which goes as $t^{1 / 7}$. Increasing $T_{2}$ is consistent with increasing vein dimensions and therefore ice crystal growth due to recrystallization processes, as was observed in the $S / V_{\mathrm{p}}$ data (Fig. 6a).

Rapid changes in $T_{2}$ values are still visible within the first 200 hours for the ice containing ECP, indicating that some microstructural rearrangement and recrystallization occurs initially after freezing. However, the $T_{2}$ values have a $\sim t^{1 / 10}$ dependence on time and the magnitude of the $T_{2}$ values at
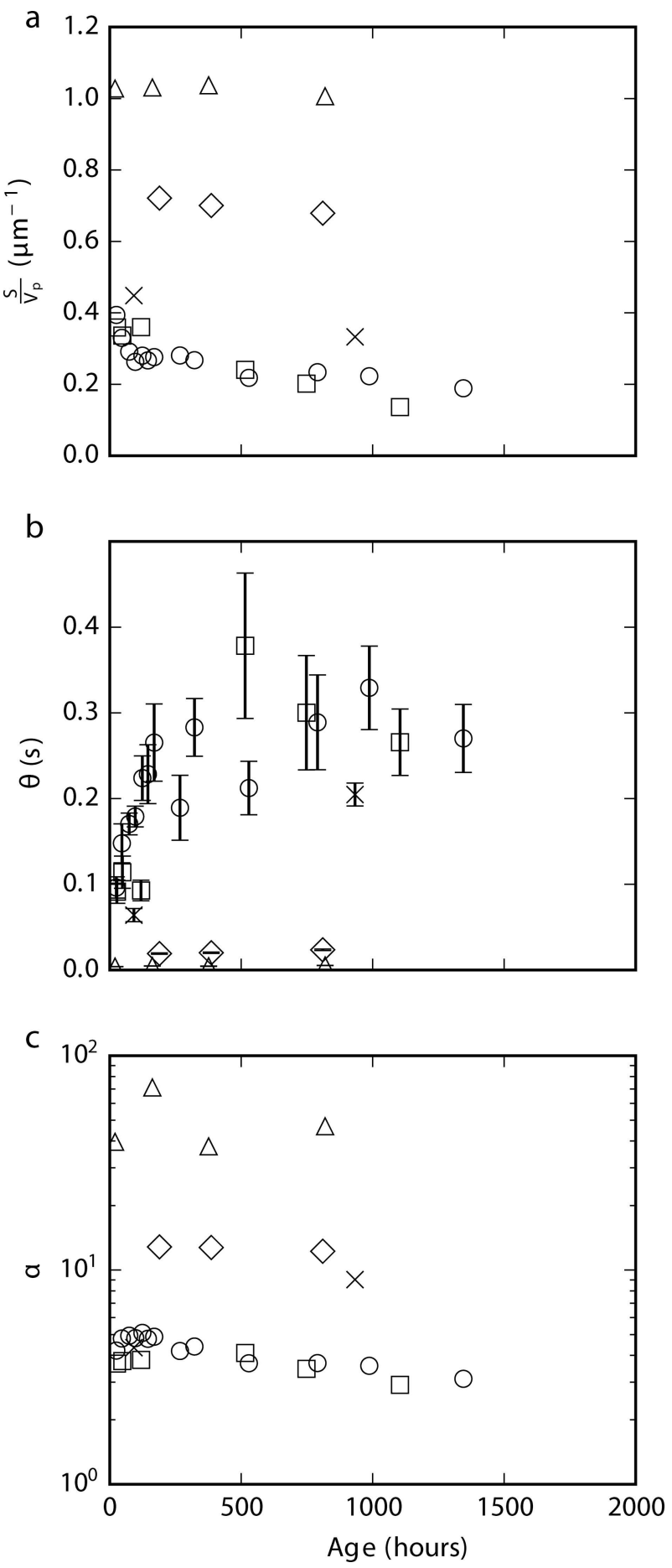

Fig. 6. Padé approximation parameters obtained from fitting the time-dependent diffusion data. Ice control (circles), ice with $10 \mu \mathrm{g} \mathrm{mL}^{-1}$ BSA (squares), ice with $10 \mu \mathrm{g} \mathrm{mL}^{-1} \mathrm{V3519-10} \mathrm{ECP}$ (crosses), ice with $2 \mu \mathrm{g} \mathrm{mL}^{-1} \mathrm{rIBP}$ (diamonds) and ice with $4 \mu \mathrm{g} \mathrm{mL}^{-1}$ rIBP (triangles). (a) Surface-to-volume ratio $S / V_{\mathrm{p}^{\prime}}$ (b) fitting parameter $\theta$ and (c) tortuosity $\alpha$. Error bars for (a) $S / V_{\mathrm{p}}$ and (c) $\alpha$ are on the order of the marker size.

longer times are half those observed in the ice controls. This indicates that the presence of ECP slows the recrystallization process, perhaps inhibiting accretion and fusing of ice crystals, resulting in smaller overall liquid grain junction dimensions at long times. The ice containing purified rIBP had $T_{2}$ values shortened by a factor of 5 and 8 for the 2 and $4 \mu \mathrm{g} \mathrm{mL}^{-1}$ concentrations, respectively, and these values remained unchanged over time. Purified rIBP therefore 

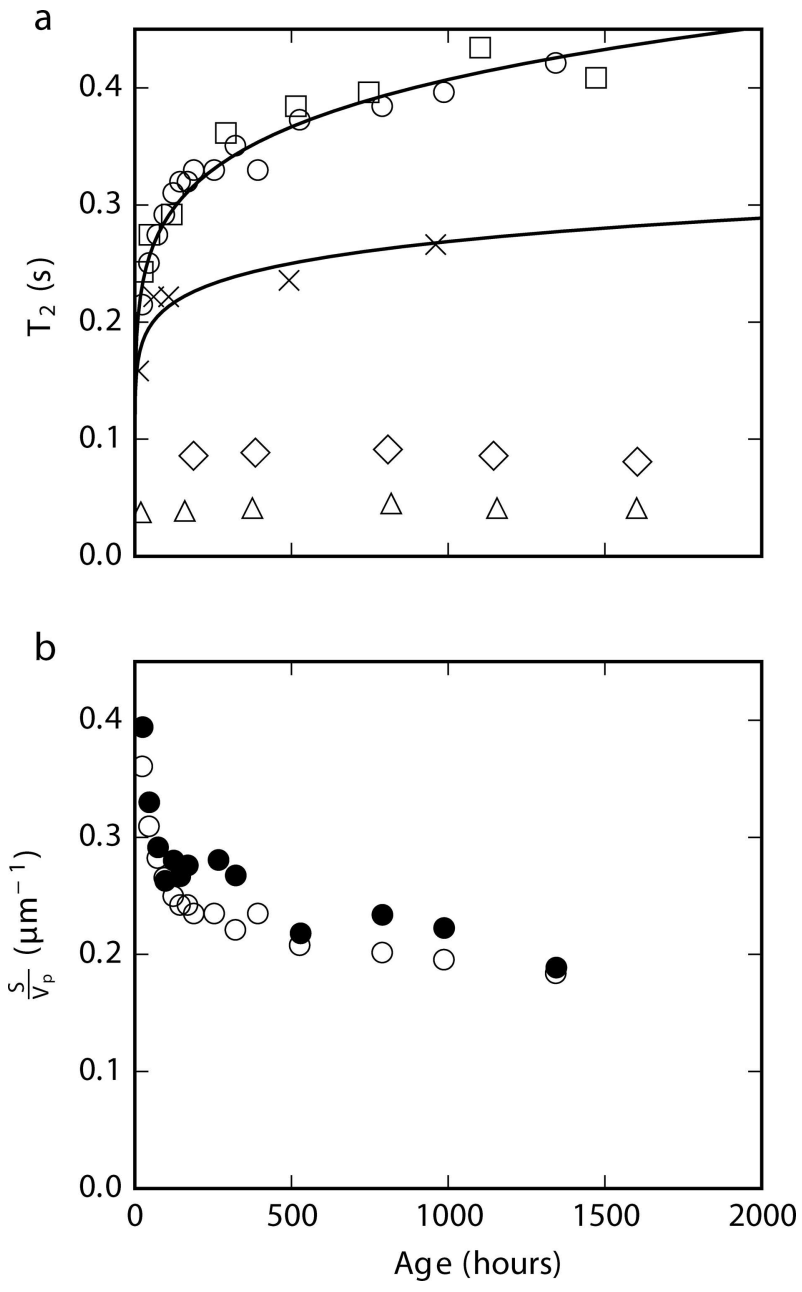

Fig. 7. (a) $T_{2}$ for all ice samples as a function of age. Ice control with $7 \mathrm{~g} \mathrm{~L}^{-1} \mathrm{NaCl}$ (circles), ice with $10 \mu \mathrm{g} \mathrm{mL}^{-1} \mathrm{BSA}$ (squares), ice with $10 \mu \mathrm{g} \mathrm{m}^{-1}$ V3519-10 ECP (crosses), ice with $2 \mu \mathrm{g} \mathrm{mL}^{-1}$ rIBP (diamonds) and ice with $4 \mu \mathrm{g} \mathrm{mL}^{-1} \mathrm{rIBP}$ (triangles). The top solid line is a power-law fit to the control data, which is proportional to $t^{1 / 7}$, while the lower solid line is a fit to the $10 \mu \mathrm{gLL}^{-1} \mathrm{~V} 3519-10$ ECP data, which is proportional to $t^{1 / 10}$. (b) $S / V_{\mathrm{p}}$ obtained from time-dependent diffusion measurements (replotted from Fig. 6a) for the ice control (filled circles) and $S / V_{p}$ obtained from scaled $1 / T_{2}$ values (open circles).

clearly inhibited growth in the liquid vein and planar junction sizes and the extent of inhibition is a function of protein concentration. Based on this, it would be reasonable to assume that the crude ECP preparation, with a $10 \mu \mathrm{g} \mathrm{mL}^{-1}$ total concentration, has an active IBP concentration $<2 \mu \mathrm{g} \mathrm{mL}{ }^{-1}$. The rapid initial change in $T_{2}$ in the ice with ECP also suggests that recrystallization occurs until coarsening reduces overall crystal numbers to the point where targets on the ice crystal prism face are saturated by IBP. For the rIBP concentrations targeted here, this occurs prior to the first measurements at 189 and 19 hours for 2 and $4 \mu \mathrm{gL}^{-1}$, respectively. Note that while the water content stays constant, indicating that the freezing process itself is complete (Fig. 4), changes in the vein network structure occur purely due to microstructural rearrangement in the ice samples from recrystallization and accretion.

The advantage of $T_{2}$ measurements is that they require only a short time to acquire $(\sim 2 \mathrm{~min}$ for the measurements reported here). A full series of time-dependent diffusion measurements (20 $\Delta$ values in this study) required $\sim 12$ hours of NMR acquisition time. However, $T_{2}$ measurements are qualitative unless the surface relaxivity $\rho$ can be determined (Eqn (1)), and surface relaxivity is a difficult physical property to measure. Regardless, when $1 / T_{2}$ is plotted versus $S / V_{p}$, the data follow a straight line with an $r$-value of 0.915 in the linear fit, indicating that $1 / T_{2}$ changes with time at the same rate as $S / V_{\mathrm{p}}$ obtained from timedependent diffusion measurements. Therefore it is possible, given a single $D(\Delta)$, to scale $1 / T_{2}$ and obtain quantitative $S / V_{p}$ (Eqn (1)). Figure $7 \mathrm{~b}$ shows $S / V_{p}$ from $D(\Delta)$ and from scaled $1 / T_{2}$ for the ice control. The agreement is good, with average percentage error of $10 \% . T_{2}$ is a useful parameter for monitoring rapid changes in ice microstructure.

\section{CONCLUSIONS}

Using novel NMR techniques we have monitored the evolution in the liquid network structure of polycrystalline ice due to recrystallization, as well as the impact of IBP activity on recrystallization processes. With increasing IBP concentration, smaller ice crystals and resultant smaller vein and planar junction dimensions were observed. This small crystal structure was found to persist over long timescales (>1600 hours). This work demonstrates the use of established porous media NMR techniques to examine the solid ice matrix and liquid-filled network formed at ice grain junctions in polycrystalline ice. As these techniques are non-invasive and non-destructive, the measurements may be used to monitor ice microstructure evolution over time. As such, there are a number of future studies that can be designed around NMR analysis of ice. A suite of factors can be used to encourage recrystallization of an ice sample (e.g. pressure, temperature), and the changes in structure can be monitored over long timescales. Similarly, natural glacial ice can be evaluated to investigate crystal structure and dimensions of the liquid-filled network. NMR studies of ice can also contribute to the study of ice kinetics within high solid fraction systems. Previous research modeling ice growth and recrystallization has been limited to crystals suspended in a salt or sugar solution so that light microscope techniques can be used to measure the change in the diameter of ice crystals (Sutton and others, 1996); however, this is not necessary with NMR.

In addition, while these measurements were performed with a high-field NMR system (5.8 T), a low-field permanent or portable NMR system could make similar measurements of $T_{2}$, signal intensity and effective diffusion to provide quantitative 3-D analysis of the liquid-filled grain junction network in polycrystalline ice. There is a current trend in development of low-field NMR hardware for specific applications, and with technological advances low-field NMR systems could be used in the field or cold rooms to process natural ice non-destructively (Callaghan and others, 1997; Hunter and others, 2009).

\section{ACKNOWLEDGEMENTS}

Funding for this project was provided by a NASA EPSCOR Research Initiation grant (J.R.B.). M.S. is partially supported by US National Science Foundation (NSF) 1204223. T.I.B. thanks Petrik Galvosas for discussions and guidance in analyzing time-dependent diffusion data, and Bing-Hao Luo, Louisiana State University, for graciously providing the rIBP. 


\section{REFERENCES}

Achberger AM, Brox TI, Skidmore ML and Christner BC (2011) Expression and partial characterization of an ice-binding protein from a bacterium isolated at a depth of 3,519 $\mathrm{m}$ in the Vostok Ice Core, Antarctica. Front. Microbiol., 2, 255 (doi: 10.3389/ fmicb.2011.00255)

Atlas RM (1995) Handbook of microbiological media for environmental microbiology. CRC Press, Boca Raton, FL

Bakermans C and Skidmore ML (2011a) Microbial metabolism in ice and brine at $-5^{\circ} \mathrm{C}$. Environ. Microbiol., 13(8), 2269-2278 (doi: 10.1111/j.1462-2920.2011.02485.x)

Bakermans $C$ and Skidmore $M(2011 b)$ Microbial respiration in ice at subzero temperatures $\left(-4^{\circ} \mathrm{C}\right.$ to $\left.-33^{\circ} \mathrm{C}\right)$. Environ. Microbiol. Rep., 3(6), 774-782 (doi: 10.1111/j.1758-2229.2011.00298.x)

Barnes PRF and Wolff EW (2004) Distribution of soluble impurities in cold glacial ice. J. Glaciol., 50(170), 311-324 (doi: 10.3189/ 172756504781829918)

Barnes PRF, Wolff E, Mallard DC and Mader HM (2003) SEM studies of the morphology and chemistry of polar ice. MicrosC. Res. Tech., 62(1), 62-69 (doi: 10.1002/jemt.10385)

Brailsford AD and Wynblatt P (1979) The dependence of Ostwald ripening kinetics on particle volume fraction. Acta Metall., 27(3), 489-497 (doi: 10.1016/0001-6160(79)90041-5)

Bray AJ (2002) Theory of phase-ordering kinetics. Adv. Phys., 51(2), 481-587 (doi: 10.1080/00018730110117433)

Brown JR, Brox TI, Vogt SJ, Seymour JD, Skidmore ML and Codd SL (2012) Magnetic resonance diffusion and relaxation characterization of water in the unfrozen vein network in polycrystalline ice and its response to microbial metabolic products. J. Magn. Reson., 225, 17-24 (doi: 10.1016/j.jmr.2012.09.011)

Brown JR and 7 others (2014) Recrystallization inhibition in ice due to ice binding protein activity detected by nuclear magnetic resonance. Biotech. Rep., 3, 60-64 (doi: 10.1016/j.btre. 2014.06.005)

Brownstein KR and Tarr CE (1979) Importance of classical diffusion in NMR studies of water in biological cells. Phys. Rev. A, 19(6), 2446-2453 (doi: 10.1103/PhysRevA.19.2446)

Callaghan PT (1991) Principles of nuclear magnetic resonance microscopy. Oxford University Press, Oxford

Callaghan PT, Coy A, MacGowan D, Packer KJ and Zelaya FO (1991) Diffraction-like effects in NMR diffusion studies of fluids in porous solids. Nature, 351(6326), 467-469 (doi: 10.1038/ 351467a0)

Callaghan PT, Eccles CD and Seymour JD (1997) An Earth's field nuclear magnetic resonance apparatus suitable for pulsed gradient spin echo measurements of self-diffusion under Antarctic conditions. Rev. Sci. Instr., 68(11), 4263-4270 (doi: 10.1063/1.1148340)

Caprihan A and Fukushima E (1990) Flow measurements by NMR. Phys. Rep., 198(4), 195-235 (doi: 10.1016/0370-1573(90) 90046-5)

Christner BC (2002) Incorporation of DNA and protein precursors into macromolecules by bacteria at $-15^{\circ} \mathrm{C}$. Appl. Environ. Microbiol., 68(12), 6435-6438 (doi: 10.1128/AEM.68.12.64356438.2002)

Christner BC (2010) Bioprospecting for microbial products that affect ice crystal formation and growth. Appl. Microbiol. Biotechnol., 85(3), 481-489 (doi: 10.1007/s00253-009-2291-2)

Christner BC, Mosley-Thompson E, Thompson LG and Reeve JN (2003) Bacterial recovery from ancient glacial ice. Environ. Microbiol., 5(5), 433-436 (doi: 10.1046/j.1462-2920.2003. 00422.x)

Cuffey KM, Conway H, Gades A, Hallet B, Raymond CF and Whitlow S (2000) Deformation properties of subfreezing glacier ice: role of crystal size, chemical impurities, and rock particles inferred from in situ measurements. J. Geophys. Res., 105(B12), 27 895-27915 (doi: 10.1029/2000JB900271)

Cullen D and Baker I (2001) Observation of impurities in ice. Microsc. Res. Tech., 55(3), 198-207 (doi: 10.1002/ jemt.10000)
Doyle S, Dieser M, Broemsen E and Christner B (2012) General characteristics of cold-adapted microorganisms. In Miller RV and Whyte LG eds Polar microbiology: life in a deep freeze. American Society for Microbiology Press, Washington, DC

Edelstein WA and Schulson EM (1991) NMR imaging of salt-water ice. J. Glaciol., 37(125), 177-180

Ewart KV, Lin Q and Hew CL (1999) Structure, function and evolution of antifreeze proteins. Cell. Mol. Life Sci., 55(2), 271-283 (doi: 10.1007/s000180050289)

Grimm RE, Stillman DE, Dec SF and Bullock MA (2008) Lowfrequency electrical properties of polycrystalline saline ice and salt hydrates. J. Phys. Chem., 112(48), 15382-15390 (doi: 10.1021/jp8055366)

Hunter MW, Dykstra R, Lim MH, Haskell TG and Callaghan PT (2009) Using Earth's field NMR to study brine content in Antarctic sea ice: comparison with salinity and temperature estimates. Appl. Magn. Reson., 36(1), 1-8 (doi: 10.1007/ s00723-009-0003-9)

Hurlimann MD, Helmer KG, Latour LL and Sotak CH (1994) Restricted diffusion in sedimentary rocks. Determination of surface-area-to-volume ratio and surface relaxivity. J. Magn. Reson. A, 111(2), 169-178 (doi: 10.1006/jmra.1994.1243)

Jepsen SM, Adams EE and Priscu JC (2006) Fuel movement along grain boundaries in ice. Cold Reg. Sci. Technol., 45(3), 158-165 (doi: 10.1016/j.coldregions.2006.05.005)

Kahlweit M (1975) Ostwald ripening of precipitates. Adv. Colloid Interface Sci., 5(1), 1-35 (doi: 10.1016/0001-8686(75) 85001-9)

Kim JS and Yethiraj A (2008) A diffusive anomaly of water in aqueous sodium chloride solutions at low temperatures. J. Phys. Chem. B, 112(6), 1729-1735 (doi: 10.1021/jp076710)

Latour LL, Mitra PP, Kleinberg RL and Sotak CH (1993) Timedependent diffusion coefficient of fluids in porous media as a probe of surface-to-volume ratio. J. Magn. Reson. A, 101(3), 342-346 (doi: 10.1006/jmra.1993.1056)

Lifshitz IM and Slyozov VV (1961) The kinetics of precipitation from supersaturated solid solutions. J. Phys. Chem. Solids, 19(1-2), 35-50 (doi: 10.1016/0022-3697(61)90054-3)

Mader HM (1992) The thermal behaviour of the water-vein system in polycrystalline ice. J. Glaciol., 38(130), 359-374

Mader HM, Pettitt ME, Wadham JL, Wolff EW and Parkes RJ (2006) Subsurface ice as a microbial habitat. Geology, 34(3), 169-172 (doi: 10.1130/G22096.1)

Mair RW and 6 others (1999) Probing porous media with gas diffusion NMR. Phys. Rev. Lett., 83(16), 3324-3327 (doi: 10.1103/PhysRevLett.83.3324)

Mair RW, Hürlimann MD, Sen PN, Schwartz LM, Patz S and Walsworth RL (2001) Tortuosity measurement and the effects of finite pulse widths on xenon gas diffusion NMR studies of porous media. Magn. Reson. Imag., 19(3-4), 345-351 (doi: 10.1016/S0730-725X(01)00247-8)

Marion GM, Mironenko MV and Roberts MW (2010) FREZCHEM: a geochemical model for cold aqueous solutions. Comput. Geosci., 36(1), 10-15 (doi: 10.1016/j.cageo.2009.06.004)

Mel'nichenko $N$ and Slobodyuk AB (2013) Nuclear magnetic resonance study of sea-water freezing mechanisms: 1 . Temperature dependence of relative brine content in sea ice. J. Glaciol., 59(216), 711-723 (doi: 10.3189/2013JoG12J239)

Menzel M, Han S, Stapf S and Blumich B (2000) NMR characterization of the pore structure and anisotropic selfdiffusion in salt water ice. J. Magn. Reson., 143(2), 376-381 (doi: 10.1006/jmre.1999.1999)

Mitra PP, Sen PN, Schwartz LM and Ledoussal P (1992) Diffusion propagator as a probe of the structure of porous media. Phys. Rev. Lett., 68(24), 3555-3558 (doi: 10.1103/PhysRevLett. 68.3555)

Mitra PP, Sen PN and Schwartz LM (1993) Short-time behavior of the diffusion coefficient as a geometrical probe of porous media. Phys. Rev. B, 47(14), 8565-8574 (doi: 10.1103/PhysRevB. 47.8565) 
Montross S and 7 others (2014) Debris-rich basal ice as a microbial habitat, Taylor Glacier, Antarctica. Geomicrobiol. J., 31(1), 76-81 (doi: 10.1080/01490451.2013.811316)

Nye JF (1989) The geometry of water veins and nodes in polycrystalline ice. J. Glaciol., 35(119), 17-22 (doi: 10.3189/ 002214389793701437)

Nye JF and Frank FC (1973) Hydrology of the intergranular veins in a temperate glacier. IASH Publ. 95 (Symposium at Cambridge 1969 - Hydrology of Glaciers), 157-161

Ohno H, Igarashi A and Hondoh T (2005) Salt inclusions in polar ice core, location and chemical form of water-soluble impurities. Earth Planet. Sci. Lett., 232(1-2), 171-178 (doi: 10.1016/ j.epsl.2005.01.001)

Price PB (2000) A habitat for psychrophiles in deep Antarctic ice. Proc. Natl Acad. Sci. USA (PNAS), 97(3), 1247-1251 (doi: 10.1073/pnas.97.3.1247)

Price WS, Ide H and Arata Y (1999) Self-diffusion of supercooled water to $238 \mathrm{~K}$ using PGSE NMR diffusion measurements. J. Phys. Chem. A, 103(4), 448-450 (doi: 10.1021/jp9839044)

Priscu JC and 9 others (1998) Perennial Antarctic lake ice: an oasis for life in a polar desert. Science, 280(5372), 2095-2098 (doi: 10.1126/science.280.5372.2095)

Priscu JC and 11 others (1999) Geomicrobiology of subglacial ice above Lake Vostok, Antarctica. Science, 286(5447), 2141-2144 (doi: 10.1126/science.286.5447.2141)

Raymond JA, Christner BC and Schuster SC (2008) A bacterial icebinding protein from the Vostok ice core. Extremophiles, 12(5), 713-717 (doi: 10.1007/s00792-008-0178-2)

Richardson C (1976) Phase relationships in sea ice as a function of temperature. J. Glaciol., 17(77), 507-519

Richardson C and Keller EE (1966) The brine content of sea ice measured with a nuclear magnetic resonance spectrometer. J. Glaciol., 6(43), 89-100

Sen PN (2004) Time-dependent diffusion coefficient as a probe of geometry. Concepts Magn. Reson. A, 23(1), 1-21 (doi: 10.1002/ cmr.a.20017)
Skidmore ML, Foght JM and Sharp MJ (2000) Microbial life beneath a high Arctic glacier. Appl. Environ. Microbiol., 66(8), 3214-3220 (doi: 10.1128/AEM.66.8.3214-3220.2000)

Song Y-Q, Cho H, Hopper T, Pomerantz AE and Sun PZ (2008) Magnetic resonance in porous media: recent progress. J. Chem. Phys., 128(5), 052212 (doi: 10.1063/1.2833581)

Stevens RN and Davies CKL (2002) Self-consistent forms of the chemical rate theory of Ostwald ripening. J. Mater. Sci., 37(4), 765-779 (doi: 10.1023/A:1013891931423)

Stillman DE, Grimm RE and Dec SF (2010) Low-frequency electrical properties of ice-silicate mixtures. J. Phys. Chem. B, 114(18), 6065-6073 (doi: 10.1021/jp9070778)

Sutton RL, Lips A, Piccirillo G and Sztehlo A (1996) Kinetics of ice recrystallization in aqueous fructose solutions. J. Food Sci., 61(4), 741-745 (doi: 0.1111/j.1365-2621.1996. tb12194.x)

Valiullin R and Furó I (2002) The morphology of coexisting liquid and frozen phases in porous materials as revealed by exchange of nuclear spin magnetization followed by ${ }^{1} \mathrm{H}$ nuclear magnetic resonance. J. Chem. Phys., 117(5), 2307-2316 (doi: 10.1063/ 1.1488585)

Voorhees PW and Glicksman ME (1984) Ostwald ripening during liquid phase sintering: effect of volume fraction on coarsening kinetics. Metall. Trans. A, 15(6), 1081-1088 (doi: 10.1063/ 1.1488585)

Wagner C (1961) Theorie der Alterung von Niederschlägen durch Umlösen (Ostwald-Reifung). Z. Elektrochem., 65(7-8), 581-591 (doi: 10.1002/bbpc.19610650704)

West JL, Rippin DM, Murray T, Mader HM and Hubbard B (2007) Dielectric permittivity measurements on ice cores: implications for interpretation of radar to yield glacial unfrozen water content. J. Environ. Eng. Geophys., 12(1), 37-45 (doi: 10.2113/JEEG12.1.37)

Zachariassen KE and Kristiansen E (2000) Ice nucleation and antinucleation in nature. Cryobiology, 41(4), 257-279 (doi: 10.1006/cryo.2000.2289) 